\title{
Finite element method for starved hydrodynamic lubrication with film separation and
} free surface effects

\author{
Poulios, Konstantinos; Vølund, Anders; Klit, Peder
}

Published in:

Computer Methods in Applied Mechanics and Engineering

Link to article, DOI:

10.1016/j.cma.2018.04.044

Publication date:

2018

Document Version

Peer reviewed version

Link back to DTU Orbit

Citation (APA):

Poulios, K., Vølund, A., \& Klit, P. (2018). Finite element method for starved hydrodynamic lubrication with film separation and free surface effects. Computer Methods in Applied Mechanics and Engineering, 339, 281-297. https://doi.org/10.1016/j.cma.2018.04.044

\section{General rights}

Copyright and moral rights for the publications made accessible in the public portal are retained by the authors and/or other copyright owners and it is a condition of accessing publications that users recognise and abide by the legal requirements associated with these rights.

- Users may download and print one copy of any publication from the public portal for the purpose of private study or research.

- You may not further distribute the material or use it for any profit-making activity or commercial gain

- You may freely distribute the URL identifying the publication in the public portal 


\section{Accepted Manuscript}

Finite element method for starved hydrodynamic lubrication with film separation and free surface effects

Konstantinos Poulios, Anders Vølund, Peder Klit

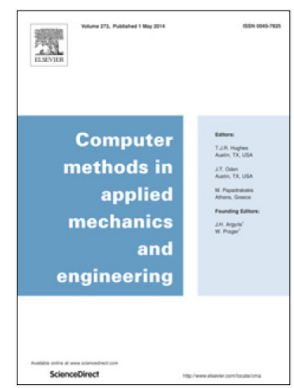

PII: $\quad$ S0045-7825(18)30229-9

DOI: $\quad$ https://doi.org/10.1016/j.cma.2018.04.044

Reference: CMA 11895

To appear in: Comput. Methods Appl. Mech. Engrg.

Received date: 22 November 2017

Revised date: 7 March 2018

Accepted date : 23 April 2018

Please cite this article as: K. Poulios, A. Vølund, P. Klit, Finite element method for starved hydrodynamic lubrication with film separation and free surface effects, Comput. Methods Appl. Mech. Engrg. (2018), https://doi.org/10.1016/j.cma.2018.04.044

This is a PDF file of an unedited manuscript that has been accepted for publication. As a service to our customers we are providing this early version of the manuscript. The manuscript will undergo copyediting, typesetting, and review of the resulting proof before it is published in its final form. Please note that during the production process errors may be discovered which could affect the content, and all legal disclaimers that apply to the journal pertain. 


\title{
Finite Element Method for Starved Hydrodynamic Lubrication with Film Separation and Free Surface Effects
}

\author{
Konstantinos Poulios* ${ }^{1}$, Anders Vølund ${ }^{2}$, and Peder Klit ${ }^{1}$ \\ ${ }^{1}$ Department of Mechanical Engineering, Technical University of Denmark, Nils Koppels \\ Allé, Building 404, 2800 Kgs. Lyngby, Denmark. \\ ${ }^{2}$ MAN Diesel \& Turbo, Teglholmsgade 41, 2450 Copenhagen SV, Denmark.
}

March 7, 2018

\begin{abstract}
This paper proposes a numerical method for determining the evolution of lubricant film thickness and pressure in partially and fully flooded regions of a hydrodynamic contact between two non-conformal rigid surfaces. The proposed method accounts for the classical Reynolds equation within the fully flooded region and for film separation with surface tension driven flow in the partially flooded region, while at the same time it resolves the a priori unknown boundary between the two regions. Additionally it deals with transitions between wetted, partially flooded regions to dry regions, where the film thickness is zero. Both pressure and film thickness fields are considered as unknowns to solve for in each time step and they are approximated through quadratic B-spline finite elements. The geometry of the gap between the rigid surfaces delimiting the lubricant is accounted for in the form of a unilateral contact condition. Appropriate complementarity conditions with respect to separation or no penetration and no slip between the lubricant and the rigid surfaces are enforced by means of a weighted residual formulation.
\end{abstract}

Keywords: hydrodynamic lubrication, surface tension, partial flooding, B-spline finite element

\section{Introduction}

The thin film and laminar flow approximate of the Navier-Stokes equations, known as Reynolds equation (Reynolds, 1886), is regarded as the fundamental equation of hydrodynamic lubrication. Hydrodynamic lubrication along with its extensions to deformable solids and thermal phenomena is relevant for a multitude of important engineering applications, which justify the extensive effort put into deriving robust and efficient numerical models based on Reynolds equation. With the treatment of fully flooded regions of a hydrodynamic contact, either with the original or with the generalized version of Reynolds equation (Dowson, 1962), being in the meanwhile rather straightforward, most of the relatively recent research efforts in this field deal with the transition to and the flow inside partially flooded regions.

In standard terminology, a fully flooded region of a hydrodynamic contact is also denoted as a pressurized zone, while partially flooded regions are normally referred to as starved or

${ }^{*}$ E-mail: kopo@mek.dtu.dk 
cavitated zones, depending on whether they are respectively located upstream or downstream of a pressurized zone. Partial flooding occurs when the available amount of lubricant is not sufficient for filling the clearance between the two solids at an acceptable pressure level and can manifest itself in different forms. In a starved inlet zone, the lubricant can either be attached to one of the surfaces or it can consist of two separate films, each attached to one of the solids. According to Dowson and Taylor (1979), different configurations are possible within a cavitated zone. Evaporation of the lubricant or release of dissolved air can result in a mixture of liquid and gas with distinct rheological properties compared to the original liquid, filling up the clearance rather homogeneously. Alternatively, the flow downstream of the pressurized zone can split into discrete finger shaped regions known as streamers, attached to both solids and separated from neighboring streamers by cavities fully occupied by gas. A third possibility, known as separation cavitation, occurs when the liquid is detached from one of the surfaces, while the remaining space in the clearance between the two solids is filled with gas. This situation is similar to a corresponding starved inlet with the lubricant film entering the contact, attached only to one of the solids. No matter whether film separation is observed upstream or downstream of the pressurized zone, the lubricant film undergoes, in such case, free surface flow governed by surface tension.

Regarding modeling of partially flooded regions in general, different approaches have been pursued depending on the specific physical system to study and the intended level of fidelity. For specific applications, the flow outside the pressurized zone can even be neglected entirely, substituted by appropriate assumptions about the transition conditions at the unknown boundary of the pressurized zone. In such cases the Reynolds equation is solved as a moving boundary problem as e.g. by Cryer (1971) and Rohde and McAllister (1975). In other cases like e.g. applications where a global mass conservation of the lubricant is of interest, partially flooded regions have to be included in the simulation. This is done e.g. by Elrod's cavitation algorithm (Elrod, 1981) and its many derivatives, which employ an additional unknown field for specifying the lubricant content in the cavitated zone in a mass conserving manner. In these cases, Couette flow is assumed in the cavitated zone corresponding to a fluid fully attached to both solids. This is a reasonable assumption when a liquid and gas mixture is formed, as is common in the cavitated zone of conformal hydrodynamic contacts, like e.g. journal bearings. But this kind of flow is unrealistic in the case of film separation, which is more common in non-conformal hydrodynamic contacts like e.g. piston ring lubrication, although it has been applied in a few instances as in (Organisciak et al., 2007) and (Zhang et al., 2016), in the lack of a better approximation. Aim of the present work is to fill this gap, providing a numerical model which combines free surface flow in partially flooded regions with the classical Reynolds equation in the pressurized zone.

Although not directly characterized as such in the popular work by Elrod, the problem of partially flooded regions in hydrodynamic contact was early recognized as a complementarity problem, (Kostreva, 1984; Strozzi, 1985). The considered contact domain is partitioned into two zones by an a priori unknown boundary, in which zones either the pressure or the content of the lubricant is prescribed, but not both at the same time. The concept of complementarity is exploited for example in (Yang and Laursen, 2009), where the problem of hydrodynamic lubrication between two deformable solids is formulated with displacement fields and a hydrodynamic pressure field as unknowns. Building upon a standard unilateral contact formulation, the aforementioned work solves the steady state Reynolds equation coupled with the equations for linearized elasticity. A similar model but extended to finite deformations is available in (Stupkiewicz et al., 2016). The latter two works are limited to steady state hydrodynamic states and the standard treatment of cavitated regions, equivalent to Elrod's algorithm, which excludes separation between 
the lubricant and the solids.

The present work deals specifically with transient flow situations and separation between the lubricant and one of the solids. This is a common situation e.g. in piston ring lubrication where one of the contact surfaces, i.e. the cylinder liner, is of much larger extent than the opposite surface, i.e. the piston ring. Lubricant supply occurs only on the liner surface where a continuous lubricant film extending over a significant area compared to the piston ring, can normally be expected. Compared to formulations based on Elrod's cavitation algorithm, that solve for the pressure and the lubricant content fields, the present work considers the lubricant film thickness (height) as the second unknown, instead of the lubricant content parameter. The full flooding condition previously expressed by the lubricant content attaining a value of one, is substituted here by the condition of the lubricant film thickness attaining a value equal to the clearance between the two solids.

The paper is structured in five sections. Following this introduction, Section 2 defines the physical system to study and the basic equations describing it. Section 3 presents the developed finite element method, which is the main contribution of this work, while Section 4 demonstrates some of the properties of the developed method with the help of numerical examples and Section 5 summarizes and concludes the paper.

\section{Problem definition}

Figure 1 defines the geometry and kinematics of two rigid solids in hydrodynamic contact as well as the geometry and kinematics of the lubricant film in the clearance between them. For the sake of simplicity the lower surface is assumed to be flat and lying in the $x y$ plane. Moreover, lubricant film separation is permitted only with respect to the upper solid. The geometry of the upper surface is described as $z=\tilde{h}(x, y, t)$, where $\tilde{h}$ can be any arbitrary continuous function of the spatial coordinates $(x, y)$ and the time variable $t$. The upper solid is considered as rigid in the sense that its shape is not affected by the forces acting on it. Nevertheless, the dependence of $\tilde{h}$ on $t$ allows for the upper surface to perform rigid body translation in directions $x, y$ and $z$ but also in the most general case to rotate and evolve in shape.

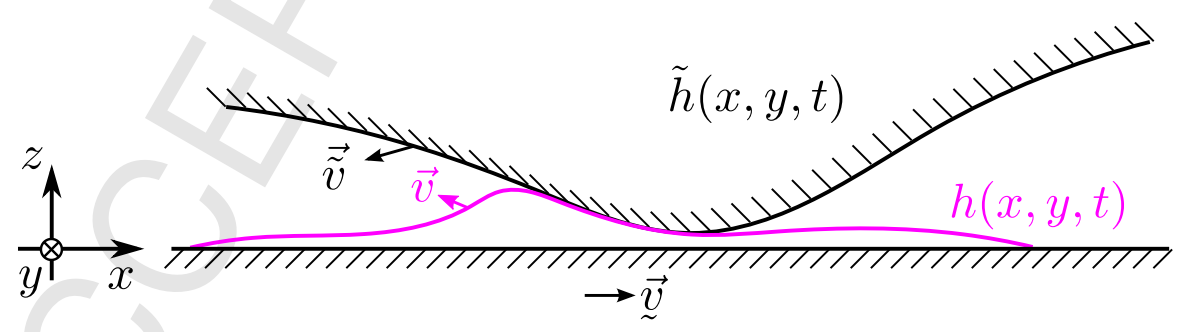

Figure 1: Geometry and kinematics of the hydrodynamic contact (quantities referring to the upper and lower solids are respectively denoted with over and under-tildes).

The kinematics of the lower and upper surfaces are respectively defined through the spatially constant vector $\underset{v}{\vec{v}}(t)$ and the vector field $\overrightarrow{\vec{v}}(x, y, t)$. In general, both $\underset{v}{\vec{v}}$ and $\overrightarrow{\vec{v}}$ can be prescribed as time dependent as indicated by the arguments adopted above. The convention that the velocity 
vector for the lower surface is parallel to the $x y$ plane can be expressed as

$$
\vec{v}(t)=\left(\begin{array}{c}
v_{x} \\
v_{y} \\
0
\end{array}\right) .
$$

At the same time there is a kinematic relationship between the time derivative of $\tilde{h}$ and the velocity field $\overrightarrow{\vec{v}}$, which describes the velocity of material points on the upper surface. This relationship is expressed through the equation

$$
\overrightarrow{\tilde{v}}(x, y, t)=\left(\begin{array}{c}
\tilde{v}_{x} \\
\tilde{v}_{y} \\
\left.\tilde{v}_{x} \frac{\partial \tilde{h}}{\partial x}+\tilde{v}_{y} \frac{\partial \tilde{h}}{\partial y}+\frac{\partial \tilde{h}}{\partial t}\right)
\end{array}\right),
$$

where $\tilde{v}_{x}(x, y, t)$ and $\tilde{v}_{y}(x, y, t)$ are the components of the vector field that can be prescribed independently of $\tilde{h}$. These components are necessary for fully defining the motion of the upper solid in order to distinguish e.g. between a rolling and a sliding cylinder.

The geometry of the lubricant film is defined through its top surface, $z=h(x, y, t)$, while its kinematics are defined through the velocity field $\vec{u}(x, y, z, t)$ for $0 \leq z \leq h$. Of special interest for some of the derivations in the sequel is the velocity of the top surface of the film defined as

$$
\vec{v}(x, y, t)=\vec{u}(x, y, h(x, y, t), t) .
$$

Similar to Eq. (2), the $z$ component of $\vec{v}$ is linked to $h(x, y, t)$ according to the expression

$$
v_{z}(x, y, t)=v_{x} \frac{\partial h}{\partial x}+v_{y} \frac{\partial h}{\partial y}+\frac{\partial h}{\partial t} .
$$

Regarding its constitutive behavior, the lubricant is assumed to be a Newtonian fluid with dynamic viscosity $\eta$, so that shear stresses at an arbitrary horizontal plane are defined as

$$
\tau_{x z}=\eta \frac{\partial u_{x}}{\partial z} \quad \text { and } \quad \tau_{y z}=\eta \frac{\partial u_{y}}{\partial z} .
$$

Additionally, under the assumptions of thin film and laminar flow, linear momentum equilibrium can be expressed with respect to a $z$ coordinate independent pressure field $p(x, y, t)$, as

$$
\frac{\partial p}{\partial x}=\frac{\partial \tau_{x z}}{\partial z} \quad \text { and } \quad \frac{\partial p}{\partial y}=\frac{\partial \tau_{y z}}{\partial z} .
$$

After substituting Eqs. (5) into Eqs. (6), the latter can be integrated twice from zero to an arbitrary coordinate $z \leq h$, whereby the integration constants can be determined in terms of the known velocity components $v_{x}$ and $v_{y}$ at the bottom and the unknown velocity components $v_{x}$ and $v_{y}$ at the top surface of the lubricant film. This treatment results in parabolic velocity profiles

$$
u_{x}=\frac{z(z-h)}{2 \eta} \frac{\partial p}{\partial x}+\frac{z}{h} v_{x}+\left(1-\frac{z}{h}\right) v_{x}
$$

and

$$
u_{y}=\frac{z(z-h)}{2 \eta} \frac{\partial p}{\partial y}+\frac{z}{h} v_{y}+\left(1-\frac{z}{h}\right) v_{y}
$$


through the film thickness.

One further integration of the velocity profiles $u_{x}$ and $u_{y}$ through the film thickness yields the corresponding net lubricant fluxes at each point $(x, y)$ as

$$
q_{x}=-\frac{h^{3}}{12 \eta} \frac{\partial p}{\partial x}+\frac{h}{2}\left(v_{x}+v_{x}\right) \quad \text { and } \quad q_{y}=-\frac{h^{3}}{12 \eta} \frac{\partial p}{\partial y}+\frac{h}{2}\left(v_{y}+v_{\sim}\right) .
$$

In order to establish a relationship between the film thickness profile $h(x, y, t)$ and the pressure profile, lubricant mass conservation has to be expressed in terms of fluxes $q_{x}$ and $q_{y}$ from Eqs. (9). Assuming an incompressible lubricant, the integral of the mass conservation equation $\nabla \cdot \vec{u}=0$ through the film thickness reads

$$
\int_{0}^{h}\left(\frac{\partial u_{x}}{\partial x}+\frac{\partial u_{y}}{\partial y}+\frac{\partial u_{z}}{\partial z}\right) d z=0 .
$$

Application of Leibniz's integral rule to Eq. (10) results in the equation

$$
\frac{\partial q_{x}}{\partial x}+\frac{\partial q_{y}}{\partial y}+v_{z}-v_{x} \frac{\partial h}{\partial x}-v_{y} \frac{\partial h}{\partial y}=0
$$

and substitution of Eqs. (4) and (9) into Eq. (11) finally yields

$$
\frac{\partial}{\partial x}\left(-\frac{h^{3}}{12 \eta} \frac{\partial p}{\partial x}+\frac{h}{2}\left(v_{x}+\underline{v}_{x}\right)\right)+\frac{\partial}{\partial y}\left(-\frac{h^{3}}{12 \eta} \frac{\partial p}{\partial y}+\frac{h}{2}\left(v_{y}+v_{y}\right)\right)+\frac{\partial h}{\partial t}=0 .
$$

The derivation of the otherwise well known transient version of Reynolds equation obtained in Eq. (12) is provided here in order to establish intermediate quantities such as the shear stresses $\tau_{x z}$ and $\tau_{y z}$, to be used later on. Reynolds Eq. (12) actually describes the relationship between the unknown fields $p, h, v_{x}$ and $v_{y}$, so that the conditions of volume conservation and linear momentum equilibrium are fulfilled. However, the problem is not fully defined until specific boundary conditions at the top surface of the film are considered.

In fully flooded regions, the top surface of the lubricant film adheres to the upper solid so that the fields $h, v_{x}$ and $v_{y}$ are prescribed as

$$
h=\tilde{h}
$$

and

$$
\left(v_{x}, v_{y}\right)=\left(\tilde{v}_{x}, \tilde{v}_{y}\right)
$$

while the pressure $p$ has to be greater than the pressure $p^{\text {fs }}$ that the lubricant would sustain if its top surface was a free surface exposed to the surrounding gas instead of being in contact with the upper solid and at the same time greater than a cavitation pressure $p^{\text {cav }}$, i.e.

$$
p>\max \left\{p^{\mathrm{fs}}, p^{\mathrm{cav}}\right\} .
$$

When $p$ drops below this limit, separation of the film and the upper solid will be assumed.

In partially flooded regions, where film separation between the lubricant and the upper solid has occurred, the fields $h, v_{x}$ and $v_{y}$ are a priori unknown and have to fulfill the conditions

$$
p=\max \left\{p^{\mathrm{fs}}, p^{\mathrm{cav}}\right\}
$$


and

$$
\left.\tau_{x z}\right|_{z=h}=\left.\tau_{y z}\right|_{z=h}=0 .
$$

along with the inequality constraint

$$
h<\tilde{h} .
$$

Eqs. (16), (17) and (18) that are valid in partially flooded regions are respectively complementary to (13), (14) and (15) that are valid in fully flooded regions. Nevertheless, the partition of the domain into partially and fully flooded subdomains is a priori unknown.

In order to complete the problem definition, the free surface pressure $p^{\text {fs }}$ has to be specified. Assuming that the surrounding gas is at an ambient pressure $p^{\mathrm{amb}}(x, y, t)$ and that the lubricant surface tension $\gamma$ is given, $p^{\mathrm{fs}}$ can be determined as a combination of $p^{\mathrm{amb}}$ and a capillary pressure. Using a low slope approximation for the curvature of the lubricant surface, for the sake of simplicity, the expression

$$
p^{\mathrm{fs}}=p^{\mathrm{amb}}-\gamma\left(\frac{\partial^{2} h}{\partial x^{2}}+\frac{\partial^{2} h}{\partial y^{2}}\right)
$$

is obtained.

After introducing an auxiliary pressure $p^{\lim }$ as

$$
p^{\lim }=\max \left\{p^{\mathrm{fs}}, p^{\mathrm{cav}}\right\},
$$

Eqs. (13), (15), (16) and (18) can be rewritten in the combined form

$$
\begin{aligned}
\tilde{h}-h & \geq 0 \\
p-p^{\lim } & \geq 0 \\
(\tilde{h}-h)\left(p-p^{\lim }\right) & =0 .
\end{aligned}
$$

The shear stresses at the top surface of the lubricant film, contained in Eqs. (17), can be evaluated based on Eqs. (5), (7) and (8) as

$$
\begin{aligned}
\left.\tau_{x z}\right|_{z=h} & =\frac{h}{2} \frac{\partial p}{\partial x}+\frac{\eta}{h}\left(v_{x}-{\underset{v}{x}}_{x}\right) \quad \text { and } \\
\left.\tau_{y z}\right|_{z=h} & =\frac{h}{2} \frac{\partial p}{\partial y}+\frac{\eta}{h}\left(v_{y}-\underline{v}_{y}\right) .
\end{aligned}
$$

Hence, Eqs. (14) and (17) can be reformulated to

$$
\left\{v_{x}, v_{y}\right\}= \begin{cases}\left\{\tilde{v}_{x}, \tilde{v}_{y}\right\} & \text { for } h=\tilde{h} \\ \left\{v_{x}, v_{y}\right\}-\frac{h^{2}}{2 \eta}\left\{\frac{\partial p}{\partial x}, \frac{\partial p}{\partial y}\right\} & \text { for } h<\tilde{h} .\end{cases}
$$

Summing up, the problem to be treated numerically in the sequel is defined in terms of:

- an initial form of the lubricant film $h(x, y, t=0)$,

- a prescribed time history of the fields $\tilde{h}, \tilde{v}_{x}, \tilde{v}_{y}, v_{x}$ and $v_{y}$ defining the solids in contact,

- a prescribed ambient pressure field $p^{\mathrm{amb}}$, 
- the viscosity $\eta$, surface tension $\gamma$ and cavitation pressure $p^{\text {cav }}$ of the lubricant and

- Eqs. (12), (21) and (23), describing the physics of the problem.

Compared to existing literature, the novel element in this description mainly concerns Eqs. (19), (20), (21) and 23) which unify two sets of equations, normally met independently for either modeling hydrodynamic lubrication or thin film free surface flow, respectively.

It should be noted that from the perspective of defining the problem, the lower solid velocity $\vec{v}$ is redundant, since any relative motion between the solids can already be expressed through the dependence of $\tilde{h}$ on $t$. From a numerical modeling perspective however, posing the problem in a way that the reference frame $x y z$ is neither bound to one solid nor the other allows for implementing both possibilities later on.

\section{$3 \quad$ Numerical Modeling}

\subsection{Dimensionless form of equations}

Before proceeding with any discretization steps, a dimensionless form of the aforementioned governing equations is introduced. A characteristic film thickness $h_{\text {ref }}$, a characteristic length $l_{\text {ref }}$ and a characteristic velocity $u_{\text {ref }}$ are some basic quantities chosen independently. A characteristic pressure is then given by

$$
p_{\text {ref }}=\frac{12 \eta u_{\text {ref }} l_{\text {ref }}}{h_{\text {ref }}^{2}}
$$

All dimensional variables employed in the previous section can be converted to their dimensionless counterparts defined as

$$
\left\{X, Y, H, T, V_{X}, V_{Y}, P\right\}=\left\{\frac{x}{l_{\mathrm{ref}}}, \frac{y}{l_{\mathrm{ref}}}, \frac{h}{h_{\mathrm{ref}}}, \frac{t u_{\mathrm{ref}}}{l_{\mathrm{ref}}}, \frac{v_{x}}{u_{\mathrm{ref}}}, \frac{v_{y}}{u_{\mathrm{ref}}}, \frac{p-p^{\mathrm{cav}}}{p_{\mathrm{ref}}}\right\} .
$$

Corresponding transformations apply to the problem data resulting in dimensionless quantities such as $\tilde{H}, \tilde{V}_{X}, \tilde{V}_{Y}, V_{X}, V_{Y}$ and $P^{\mathrm{amb}}$. Moreover, a dimensionless surface tension parameter

$$
\Gamma=\frac{\gamma}{\gamma_{\mathrm{ref}}},
$$

is introduced, where

$$
\gamma_{\mathrm{ref}}=\frac{12 \eta u_{\mathrm{ref}} l_{\mathrm{ref}}^{3}}{h_{\mathrm{ref}}^{3}}
$$

In order to enable a more compact notation in the sequel, the vectors

$$
\vec{V}=\left(V_{X}, V_{Y}\right)^{T}, \quad \overrightarrow{\tilde{V}}=\left(\tilde{V}_{X}, \tilde{V}_{Y}\right)^{T} \quad \text { and } \quad \underset{\sim}{\vec{V}}=(\underbrace{}_{X}, V_{Y})^{T}
$$

as well as the gradient operator

$$
\vec{\nabla}=(\partial / \partial X, \partial / \partial Y)^{T}
$$

are introduced.

Based on the dimensionless variables and problem data introduced above, Eq. (20) can be expressed in terms of dimensionless quantities as

$$
P^{\lim }=\left[P^{\mathrm{amb}}-\Gamma \nabla^{2} H\right]_{+},
$$


where []$_{+}$is the positive part operator, also known as Macaulay operator.

Respectively, Eq. (12), (21) and (23) translate to

$$
\begin{aligned}
\vec{\nabla} \cdot\left(-H^{3} \vec{\nabla} P+\frac{H}{2}(\vec{V}+\vec{V})\right) & +\frac{\partial H}{\partial T}=0, \\
\tilde{H}-H & \geq 0 \\
P-P^{\lim } & \geq 0 \\
(\tilde{H}-H)\left(P-P^{\lim }\right) & =0
\end{aligned}
$$

and

$$
\vec{V}= \begin{cases}\overrightarrow{\tilde{V}} & \text { for } H=\tilde{H} \\ \vec{V}-6 H^{2} \vec{\nabla} P & \text { for } H<\tilde{H}\end{cases}
$$

\subsection{Discretization}

Eqs. (31), (32) and (33) form a system of equations for $H, P, V_{X}$ and $V_{Y}$. By substituting the third of these three equations into the first one, it is possible to eliminate $V_{X}$ and $V_{Y}$ and obtain a system for $H$ and $P$ only. However, one should observe the strong discontinuity at the border between the two regimes of Eq. (33) that results in a non-differentiable system. Hence, a regularization of this discontinuity is performed here by substituting Eq. (33) with

$$
\vec{V} \approx \xi \overrightarrow{\vec{V}}+(1-\xi)\left(\vec{V}-6 H^{2} \vec{\nabla} P\right)
$$

where

$$
\xi=\left\{\begin{array}{lll}
0 & \text { for } \quad H \leq \tilde{H}-H_{\text {th }} \\
1-\frac{\tilde{H}-H}{H_{\text {th }}} & \text { for } \quad \tilde{H}-H_{\text {th }}<H<\tilde{H} \\
1 & \text { for } \quad H \geq \tilde{H} .
\end{array}\right.
$$

This regularization postulates some interaction between the upper solid and the lubricant top surface when the gap between both is smaller than the threshold thickness $H_{\mathrm{th}}$, which serves as the regularization parameter. Within this range, the velocity of the top surface of the lubricant film is provided by a linear interpolation between the traction free condition velocity and the upper solid velocity. Although it is not the main motivation for this treatment, a physical interpretation exists and can actually provide a reference for choosing appropriate values for $H_{\mathrm{th}}$. Considering a surface roughness for the upper solid, asperity peaks can interact with the lubricant top surface before the lubricant is fully attached. In such situations, neither the velocity of the lubricant top surface is equal to that of the upper solid, nor the surface traction is zero, but an intermediate situation can be expected as the one expressed by Eq. (34). Hence, the dimensionless parameter $H_{\text {th }}$ can be chosen as the ratio of the upper surface roughness to the reference thickness $h_{\text {ref }}$.

The regularized velocity $\vec{V}$ according to Eqs. (34) and (35) is piecewise differentiable with respect to $H$ and substitution of Eq. (34) into Eq. (31) results in the equation

$$
\vec{\nabla} \cdot\left(\xi \frac{H}{2} \overrightarrow{\vec{V}}+(2-\xi) \frac{H}{2} \underset{V}{\vec{V}}+(3 \xi-4) H^{3} \vec{\nabla} P\right)+\frac{\partial H}{\partial T}=0,
$$

which is also piecewise differentiable if $\xi$ is according to Eq. (35). 
The next step is to provide an alternative formulation for Eq. (32) that is free of inequality constraints. Obviously, the condition

$$
\min \left\{\tilde{H}-H, \frac{P-P^{\lim }}{r}\right\}=0
$$

holds only if one of the two arguments of the min function is equal to zero and the other argument is non-negative, which for any positive constant $r$ is equivalent to Eq. (32). The positive parameter $r$ is a scaling coefficient, equivalent to the augmentation parameter in the corresponding formulation in contact mechanics by Curnier and Alart (1988). Its value is insignificant in the continuous setting but $r$ can affect the distribution of the discretization error among the $H$ and $P$ fields in the discrete setting.

In order to solve the system of piecewise differentiable Eqs. (36) and (37) for the unknown fields $H$ and $P$ numerically, discrete approximations $H_{h}$ and $P_{h}$ from the finite element spaces $\mathcal{V}_{H}^{h}$ and $\mathcal{V}_{P}^{h}$ are considered for $H$ and $P$ respectively. Moreover, a first order backward Euler approximation of the time derivative in Eq. (36) is employed, based on the known film thickness field $H_{h 0}$ at the previous time instant. A weighted residual enforcement of the considered set of equations can be obtained with the help of test functions $q$ and $w$ in the weak form

$$
\int_{\Omega}\left(\vec{\nabla} \cdot\left(\xi \frac{H_{h}}{2} \overrightarrow{\tilde{V}}+(2-\xi) \frac{H_{h}}{2} \vec{V}+(3 \xi-4) H_{h}^{3} \vec{\nabla} P_{h}\right)+\frac{H_{h}-H_{h 0}}{\Delta T}\right) q d \Omega=0 \quad \forall q \in \mathcal{V}_{q}^{h}
$$

and

$$
\int_{\Omega} \min \left\{\tilde{H}-H_{h}, \frac{P_{h}-\left[P^{\mathrm{amb}}-\Gamma \nabla^{2} H_{h}\right]_{+}}{r}\right\} w d \Omega=0 \quad \forall w \in \mathcal{V}_{w}^{h},
$$

where $\Omega$ is the two dimensional computational domain in the $X Y$ space and $\mathcal{V}_{q}^{h}$ and $\mathcal{V}_{w}^{h}$ are appropriate finite element spaces. It is noted that $\xi$ in Eq. (38) is a function of the unknown field $H_{h}$ according to Eq. (35).

There are different possibilities for choosing the finite element spaces for the discrete approximations $H_{h}$ and $P_{h}$ as well as for the discrete test functions $q$ and $w$. The simplest choice is to use one common set of basis functions to construct all four spaces. Assuming also for the sake of simplicity that both fields $H$ and $P$ are prescribed along the whole boundary $\partial \Omega$ of the computational domain $\Omega$, the corresponding finite element spaces $\mathcal{V}_{H}^{h}$ and $\mathcal{V}_{P}^{h}$ have to be compatible with these boundary conditions, while the finite element spaces $\mathcal{V}_{q}^{h}$ and $\mathcal{V}_{w}^{h}$ for the test functions have to be homogeneous along $\partial \Omega$.

For test functions of this kind, integration of Eq. (38) by parts results in

$$
\int_{\Omega}-\left(\xi \frac{H_{h}}{2} \overrightarrow{\tilde{V}}+(2-\xi) \frac{H_{h}}{2} \vec{V}+(3 \xi-4) H_{h}^{3} \vec{\nabla} P_{h}\right) \cdot \nabla q+\frac{H_{h}-H_{h 0}}{\Delta T} q d \Omega=0 \quad \forall q \in \mathcal{V}_{q}^{h},
$$

whereas the non-smooth min operator in Eq. 39 does not allow a similar treatment for reducing the order of the Laplacian operator on $H_{h}$. As a consequence, Eq. 39 requires a $\mathrm{C}^{1}$ continuous approximation $H_{h}$ of $H$.

In the present work, quadratic B-spline basis functions are used to construct all four spaces $\mathcal{V}_{H}^{h}, \mathcal{V}_{P}^{h}, \mathcal{V}_{q}^{h}$ and $\mathcal{V}_{w}^{h}$ with $\mathrm{C}^{1}$ continuity on Cartesian grids in one or two dimensions. In accordance with the polynomial order of the adopted basis functions, numerical integration is performed with three Gauss points per element in one dimension and nine Gauss points per quadrilateral element 
in two dimensions. A useful extension not included in the present work would be the use of a hierarchical refinement strategy of the employed uniform B-spline spaces according to Bornemann and Cirak (2013).

A generalized Newton scheme is used for the final solution of the semi-smooth system of discretized Eqs. (39) and (40), combined with Eq. (35). The linearization of the aforementioned equations for deriving the corresponding stiffness matrix is performed by means of symbolic differentiation within the finite element framework GetFEM++, (Pommier and Renard, 2017). Constant time steps are assumed for all simulations presented in the next section with the additional possibility of subdividing a time step upon failure of the Newton algorithm to converge.

\subsection{Treatment of contact lines and stabilization}

In the partially flooded region, the sought solution for the film thickness field $H$ corresponds to the so called lubrication approximation of thin film free surface flow, described in strong form by the equation

$$
\underset{\vec{V}}{\vec{V}} \cdot \vec{\nabla} H+\Gamma \vec{\nabla} \cdot\left(H^{3} \vec{\nabla}\left(\nabla^{2} H\right)\right)+\frac{\partial H}{\partial T}=0,
$$

which is recovered from Eq. (36) for $\xi=0$ and $P=P^{\text {lim }}$, with $P^{l i m}$ from Eq. (30), after neglecting the non-negativity constraint on $P^{\text {lim }}$.

The evolution Eq. (41) has an advection term due to a possibly nonzero velocity $\vec{\sim}$ with respect to the chosen frame of reference and a fourth order degenerate diffusion term associated with the surface tension parameter $\Gamma$. For positive $H$, the presence of the diffusion term results in a smooth solution, which can be approximated in a finite element space efficiently. In the limit $H \rightarrow 0$ however, the diffusion effect of the surface tension $\Gamma$ vanishes. Especially, for a cubic film thickness factor as in Eq. (41), the latter is not positivity preserving, not even in the continuous setting, (Bertozzi, 1998). As a consequence, the discretized formulation presented in the previous section actually results in non-physical oscillatory and partially negative solutions, if left untreated.

The boundaries between wetted regions, where $H>0$, and dry regions, where $H=0$, are known as wetting or contact lines and the actual film thickness evolution at moving contact lines has been studied extensively, (de Gennes, 1985). Figure 2 illustrates how in reality, the transition from a developed film to a dry region can be rather complex, involving multiple size scales where different physics dominate the lubricant flow. This is for example the case for so called precursor films, shown left in the figure. Resolving the actual lubricant flow at lower size scales is beyond the scope of the present work, which focuses on providing a numerical scheme that remains stable despite the sharp transitions in the vicinity of contact lines, as illustrated on the right hand side of Figure 2.
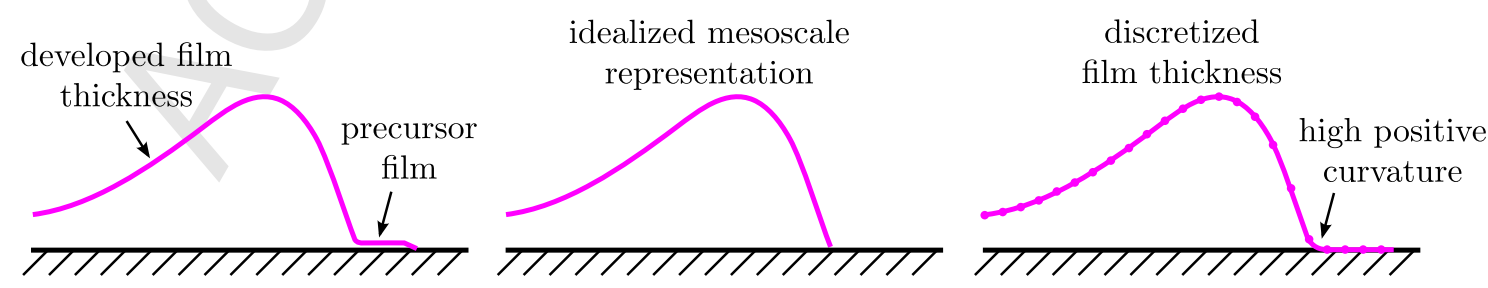

Figure 2: Simplified physics at liquid contact lines. 
One possibility for dealing with this kind of sharp transitions is to consider available discontinuity capturing methods from the literature. However, despite the plethora of proposed schemes for the classical advection-diffusion equation in the context of Lagrange finite elements, there is a lack of corresponding treatments for the fourth order diffusion term of the present work, especially in combination with $\mathrm{C}^{1}$ continuous elements. For linear elements, a relevant regularization for preserving positivity based on an entropy dissipating scheme has been proposed in (Zhornitskaya and Bertozzi, 2000; Bertozzi and Bowen, 2002).

Before introducing the regularization implied in Figure 2, it is essential to observe that the high positive curvature at the contact line, on the right hand side of the figure, is simply a feature of the discretization. The curvature at this point is actually expected to be mesh dependent and not linked to any underlying physics and the actual film form. Therefore, the high curvature at the contact line has to be decoupled from the capillary pressure term, appearing e.g. in Eq. (39) To this end, the term $\Gamma \nabla^{2} H$ will be substituted by the term

$$
c(H, \nabla H) \Gamma \nabla^{2} H,
$$

where the cutoff function $c(H, \nabla H)$ has to be defined to be equal to one in regions of a fully developed film and approximately zero close to the contact line and in dry regions $(H \rightarrow 0)$.

A second component of the proposed regularization is an enforcement of a non-negativity condition. One could penalize negative values or use Lagrange multipliers for constraining the film thickness field to non-negative values but such a treatment would annul the volume conserving structure of Eq. (41). Eq. (41), similar to Eq. (36), is an evolution equation with respect to $H$ in the form

$$
\vec{\nabla} \cdot \vec{Q}+\frac{\partial H}{\partial T}=0
$$

which intrinsically preserves the volume between the zero plane and the surface $H(X, Y)$, for any arbitrary flow field $\vec{Q}(X, Y)$. The basic idea for enforcing non-negativity here is to consider an appropriate additional flow $\vec{Q}_{\mathrm{cl}}(X, Y)$ in the vicinity of contact lines.

Limiting all further derivations to the one-dimensional case for the sake of simplicity and considering an arbitrary point $X$ within an element $e$ close to a contact line, the film thickness at a distance $\Delta X$ ahead of the considered point can be estimated by the expression

$$
H_{h}(X+\Delta X) \approx H_{h}(X)+\Delta X H_{h}^{\prime}(X),
$$

where $H_{h}^{\prime}$ is simply the spatial derivative of $H_{h}$ in $X$ direction.

For $|\Delta X|$ close to the element size $l_{e}$, Eq. (44) approximates the film thickness in the neighboring element. By defining an additional flow $Q_{\mathrm{cl}}$ proportional to the negative part of the approximated film thickness ahead of the considered point, non-negativity of the film thickness can actually be preserved. The direction of $Q_{\mathrm{cl}}$ is chosen to work against the negative part of $H+\Delta X H^{\prime}$, hence, if $Q_{\mathrm{cl}}$ is sufficiently scaled compared to all other terms, it will diminish the quantity $H+\Delta X H^{\prime}$. In the limit of this quantity becoming zero, it is easy to show that the film thickness close to the contact line attains the form of an exponential decay, cf. Figure 3.

The method can be generalized to apply to both positive and negative slopes $H^{\prime}$, leading to the following expression for the additional flow term

$$
Q_{\mathrm{cl}}=w_{\mathrm{cl}} \min \left\{\max \left\{-H_{h}-\delta_{\mathrm{cl}} l_{e} H_{h}^{\prime}, 0\right\}, H_{h}-\delta_{\mathrm{cl}} l_{e} H_{h}^{\prime}\right\},
$$

where $w_{\mathrm{cl}}$ is an overall scaling coefficient and $\delta_{\mathrm{cl}}$ is a parameter, close to one, defining the length $\Delta X$ as a fraction of the element length $l_{e}$. 
Although the proposed regularization is motivated by the fluid flow physics underlying Eq. (43), it can possibly be used for preserving non-negativity in a much broader context. An extension to two dimensional domains, especially for Cartesian meshes, should also be rather straightforward. This also includes non square elements, where the employed stabilization terms would simply need to account for two different element sizes $l_{e}$ along each of the Cartesian axes.

It should be reminded here that although the regularization $Q_{\mathrm{cl}}$ leads to a smooth transition to zero film thickness, this transition does not represent the actual film form and it should not be interpreted e.g. in terms of a wetting angle. The actual contribution of the proposed regularization is to avoid pollution of the finite element approximation with a spatially and temporally oscillating error, achieving that in the least intrusive manner. But beyond that, free surface flow physics still have to be turned off in a region at least as large as the regularized region, as explained earlier with respect to Eq. (42).

The aforestated derivation of $Q_{\mathrm{cl}}$ motivates also the definition of a cutoff term $c\left(H, H^{\prime}\right)$ for Eq. (42). In case of $Q_{\mathrm{cl}}$, the negative part of $H+\Delta X H^{\prime}$ was used as a measure of proximity to a contact line, for defining an additional regularizing flow, with $\Delta X$ in the order of an element size. Regarding the capillary pressure cutoff function $c\left(H, H^{\prime}\right)$, the same idea can be employed. In this case however, the length scale $\Delta X$ has to be mesh independent and actually larger than the length $\delta_{\mathrm{cl}} l_{e}$ used in the definition of $Q_{\mathrm{cl}}$. The final expression for $c\left(H, H^{\prime}\right)$, proposed and tested numerically in the present work is

$$
c\left(H, H^{\prime}\right)=\min \left\{1, \frac{\left[H-\rho_{\mathrm{cl}}\left|H^{\prime}\right|\right]_{+}}{\rho_{\mathrm{cl}}}\right\}
$$

which is valid both for positive and negative slopes $H^{\prime}$ with $\rho_{\mathrm{cl}}$ being a length scale parameter. Increasing $\rho_{\mathrm{cl}}$ leads to a larger capillarity cutoff region near contact lines. Considering $\rho_{\mathrm{cl}}$ as an independent parameter and $\delta_{\mathrm{cl}}$ as a finite element formulation constant, the condition $\rho_{\mathrm{cl}}>\delta_{\mathrm{cl}} l_{e}$ can be used to estimate the necessary element size $l_{e}<\rho_{\mathrm{cl}} / \delta_{\mathrm{cl}}$ for achieving mesh convergence. Numerical experiments show that element sizes of approximately half of the size predicted by the latter condition lead to satisfactory convergence.

Last, in case of a nonzero advective velocity $\vec{\sim}$, extra stabilization is necessary with respect to the advection term in Eq. (41) such as the streamline upwind Petrov-Galerkin (SUPG) method. The SUPG stabilization term is linear, however, its sign and magnitude depend on the advective velocity. For the $\mathrm{C}^{1}$ continuous finite element spaces employed in the present work an alternative stabilization scheme is proposed here, based on a nonlinear stabilization term. The basic idea 
comes from applying the concept of Figure 3 to $H^{\prime}$ instead of $H$. Any spurious oscillations due to a numerical instability are characterized by rapid transitions of $H^{\prime}$ through zero. Controlling the rate at which $H^{\prime}$ can approach zero, will automatically suppress any such spurious modes in the solution. One difference compared to Figure 3 is that $H^{\prime}$ can approach the zero level both from positive and negative values. Accounting for all possible combinations of signs between $H^{\prime}$ and $H^{\prime \prime}$ results in the following fourth order nonlinear diffusion term in a weak form,

$$
w_{s t a b}\left(\left[\left|H^{\prime}\right|-\delta_{\mathrm{cl}} l_{e} H^{\prime \prime}\right]_{-}-\left[\left|H^{\prime}\right|+\delta_{\mathrm{cl}} l_{e} H^{\prime \prime}\right]_{-}\right) q^{\prime \prime}
$$

where $q^{\prime \prime}$ is the second spatial derivative of the test functions $q$ from Eq. (40). As in the case of Eq. (45), the stabilization term of Eq. (47) has to be scaled appropriately with a weight $w_{\text {stab }}$. Numerical tests in the following section will demonstrate that the solution is rather insensitive to the choice of $w_{\mathrm{cl}}$ and $w_{\text {stab }}$, within a wide range of values.

The final set of equations to solve are derived from Eqs. (39) and (40). For this purpose, the term $Q_{\mathrm{cl}} q^{\prime}$ with $Q_{\mathrm{cl}}$ from Eq. (45) as well as the stabilization term from Eq. (47) are added into the integral of Eq. (40). At the same time, the capillary pressure $\Gamma H^{\prime \prime}$ in Eq. (39) is substituted by its regularized version according to Eq. (42), with the cutoff function defined in Eq. (46).

\section{$4 \quad$ Numerical studies}

This section presents numerical results from one dimensional simulations of a slider (upper solid) displacing a patch of lubricant with an initially known film thickness profile, attached on a substrate (lower surface). The initial configuration of the slider and the lubricant film is shown in Figure 4, defined in terms of the dimensionless quantities $X$ and $H$ within the computational domain $\Omega: X \in[0,30]$. The lower surface is moving at a dimensionless speed $V_{X}=1$ with respect to the computational domain, while the slider is fixed in the $X$ direction. In order to simplify the problem, instead of the Dirichlet boundary conditions assumed in section 3.2, periodic boundary conditions are assumed here both for the lubricant film thickness $H(X)$ and the pressure field $P(X)$, resulting in $H(0)=H(30)$ and $P(0)=P(30)$ respectively. These boundary conditions are implemented by choosing corresponding periodic spaces $\mathcal{V}_{H}^{h}, \mathcal{V}_{P}^{h}, \mathcal{V}_{q}^{h}$ and $\mathcal{V}_{w}^{h}$. Due to the $\mathrm{C}^{1}$ continuity of the employed approximation, equality of derivatives between the ends of the domain is also automatically imposed, i.e. $H^{\prime}(0)=H^{\prime}(30)$ and $P^{\prime}(0)=P^{\prime}(30)$.

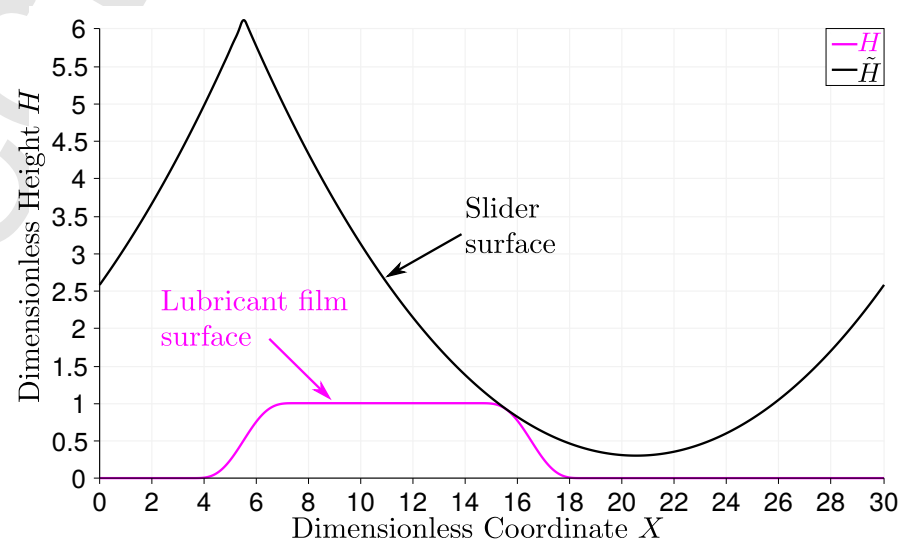

Figure 4: Initial lubricant film shape and slider geometry and position. 
The initial film thickness profile illustrated in Figure 4 is mathematically defined as

$$
H(X)=1-\mathcal{S}\left(\frac{|X-11|-3.5}{4}\right)
$$

where $\mathcal{S}$ is the seventh degree smooth step function

$$
\mathcal{S}(x)= \begin{cases}0 & \text { for } x<0 \\ -20 x^{7}+70 x^{6}-84 x^{5}+35 x^{4} & \text { for } 0 \leq x \leq 1 \\ 1 & \text { for } x>1\end{cases}
$$

A circular slider of radius $R$ in physical space is transformed into an elliptical geometry in terms of the dimensionless coordinates $X$ and $H$, defined by the expression

$$
\tilde{H}(X)=G+R_{H}\left(1-\sqrt{\left[1-\left(\frac{X-20.52}{R_{X}}\right)^{2}\right]_{+}}\right),
$$

where $R_{H}=R / h_{\text {ref }}$ and $R_{X}=R / l_{\text {ref }}$, while $G$ represents the minimum gap between the slider and the substrate. In order to account for a periodic array of sliders, as indicated in Figure $4, \tilde{H}$ can be substituted by an expression like $\min \{\tilde{H}(X), \tilde{H}(X+30)\}$ instead.

The numerical results shown in this section correspond to the slider geometry of Figure 4 with $R_{H}=80$ and $R_{X}=40$ and an initial gap $G=0.3$. The dimensionless ambient pressure $P^{\mathrm{amb}}$ is kept fixed at 0.5 and a surface tension parameter $\Gamma=0.1$ is considered, except for a comparison with $\Gamma=0.01$, provided in subsection 4.2. Table 1 summarizes a set of independent and derived scaling parameters that can be used in order to link the dimensionless problem parameters to their dimensional counterparts describing the problem in the physical space. Based on these reference values, the investigated example corresponds to a circular slider with a radius of $80 \mathrm{~mm}$ and a clearance of $0.3 \mathrm{~mm}$ from the substrate surface, sweeping an approximately $20 \mathrm{~mm}$ wide and $1 \mathrm{~mm}$ thick droplet, attached on the substrate. The dynamic viscosity of the droplet fluid is the one given in Table 1 and its surface tension is $4.8 \cdot 10^{-4} \mathrm{~N} / \mathrm{mm}$, for $\Gamma=0.1$.

\begin{tabular}{lccc}
\hline Fluid dynamic viscosity & $\eta$ & $10^{-8}$ & {$\left[\mathrm{~N} \mathrm{~s} / \mathrm{mm}^{2}\right]$} \\
Reference film thickness & $h_{\text {ref }}$ & 1 & {$[\mathrm{~mm}]$} \\
Reference length & $l_{\text {ref }}$ & 2 & {$[\mathrm{~mm}]$} \\
Reference velocity & $u_{\text {ref }}$ & $5 \cdot 10^{3}$ & {$[\mathrm{~mm} / \mathrm{s}]$} \\
\hline Reference pressure (derived) & $p_{\text {ref }}$ & $1.2 \cdot 10^{-3}$ & {$\left[\mathrm{~N} / \mathrm{mm}^{2}\right]$} \\
Reference suurface tension (derived) & $\gamma_{\text {ref }}$ & $4.8 \cdot 10^{-3}$ & {$[\mathrm{~N} / \mathrm{mm}]$} \\
\hline
\end{tabular}

Table 1: Reference quantities for converting between dimensional and dimensionless parameters.

\subsection{Impact of numerical parameters}

Numerical results shown in this section are based on the modified versions of Eqs. (39) and (40) proposed in section 3.3. A series of numerical studies investigate the impact of the regularization parameter $H_{\text {th }}$ in Eq. (35), the parameter $r$ in Eq. (39), the scaling parameters $w_{\mathrm{cl}}$ and $w_{\text {stab }}$ in Eqs. (45) and (47) as well as the parameter $\rho_{\mathrm{cl}}$ in Eq. (46). To this end, simulations are performed 
with a total of 550 time steps of size $\Delta T=0.05$ and the gap $G=0.3$ being prescribed not only as an initial value, but during the complete simulation.

The value of the parameter $\delta_{\mathrm{cl}}$ in Eqs. (45) and (47) is not part of this study because it is advantageous to define it as low as possible. Numerical experiments have shown that a value $\delta_{\mathrm{cl}}=0.75$ is close to the sharpest exponential decay $\mathrm{e}^{-X /\left(\delta_{\mathrm{cl}} l_{e}\right)}$, that quadratic B-spline finite elements can capture without any significant oscillatory error component. Hence, this value was adopted for all numerical studies throughout the present work.

Figure 5 shows numerical solutions regarding the film thickness and pressure field at the 300th time step for three different meshes, with element sizes from 0.05 to 0.2 . The intermediate mesh with 300 elements delivers a solution practically equivalent to the one from the finest mesh and the majority of the remaining studies were performed with this intermediate mesh.

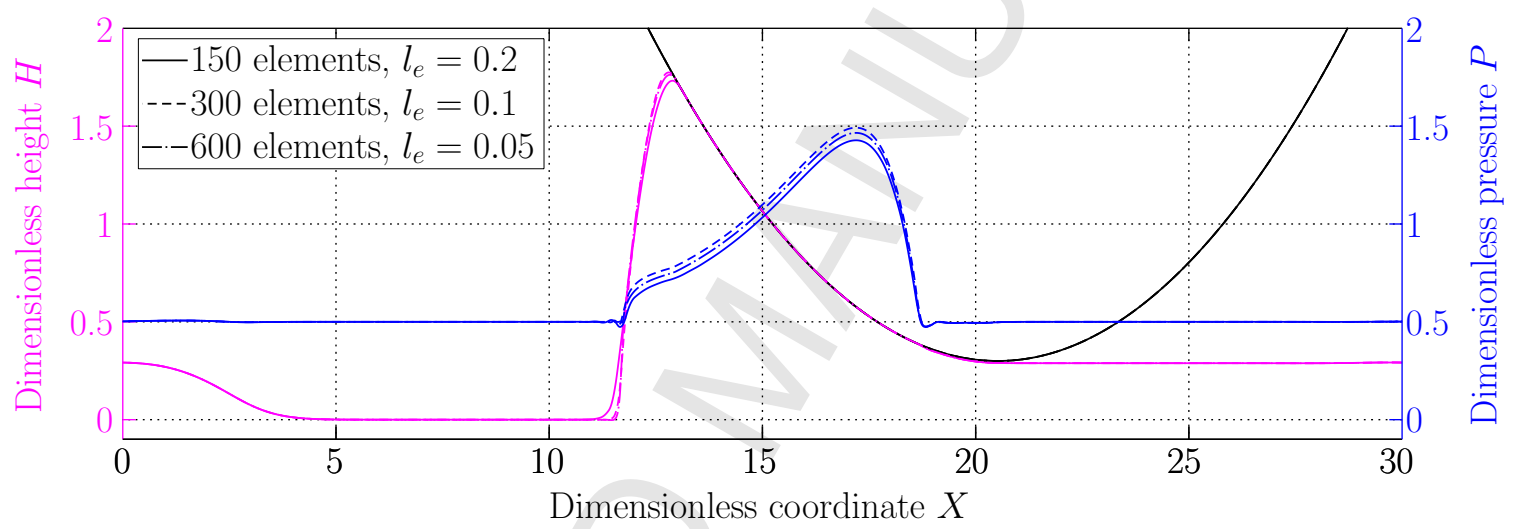

Figure 5: Calculated film thickness and pressure profiles at time $T=15$, for $\Gamma=0.1, H_{\mathrm{th}}=0.01$, $w_{\mathrm{cl}}=10, w_{\mathrm{stab}}=0.1, \rho_{\mathrm{cl}}=0.2, r=10$.

Figure 6 illustrates the sensitivity of the solution on the numerical parameter $r$, showing the pressure profile at the region where the hydrodynamic pressure hill ends and the lubricant film separates from the upper solid, which is the part of the solution that is affected most. Already for the coarsest mesh, the impact of $r$ is rather moderate, while for the intermediate mesh the differences between the three provided curves are insignificant. All further results are obtained with the intermediate $r$ value of 10 .

Regarding the non-negativity regularization with the additional flow term from Eq. (45), a specific choice of $\delta_{\mathrm{cl}}$ has been justified above. However, the overall scaling parameter $w_{\mathrm{cl}}$ can be chosen rather arbitrarily. Figure 7 provides some hint regarding the range of appropriate values for $w_{\mathrm{cl}}$ by comparing calculated thickness profiles for $w_{\mathrm{cl}}=10$ and $w_{\mathrm{cl}}=1000$. Naturally, differences occur only at the location where the film thickness $H$ transitions to zero, hence the figure is limited to that region. Looking at the logarithmic version of the graph on the right hand side of the figure, it becomes evident that a high value of $w_{\mathrm{cl}}=1000$ is necessary to approximate the exponential decay function anticipated in Figure 3. For practical purposes though, the transition obtained with $w_{\mathrm{cl}}=10$ is satisfactory and this value is used for the remaining studies of this section. It should be noted that neither $w_{\mathrm{cl}}$ nor $\delta_{\mathrm{cl}}$ affect the physics of the problem, keeping in mind of course that $\delta_{\mathrm{cl}}$ is by definition always close to unity. The obtained film thickness and pressure curves for the cases shown in Figure 7 are otherwise indistinguishable apart from the sharp transition location included in the figure.

Figure 8 shows how the scaling $w_{\text {stab }}$ of the stabilization term of Eq. (47) affects the solution 
150 elements

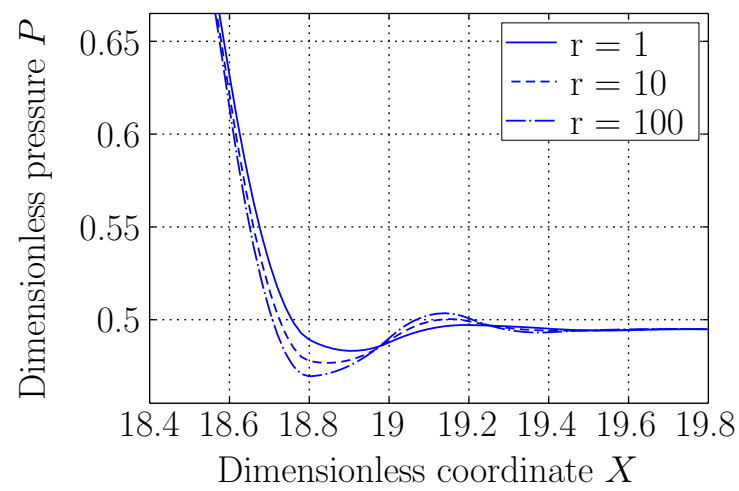

300 elements

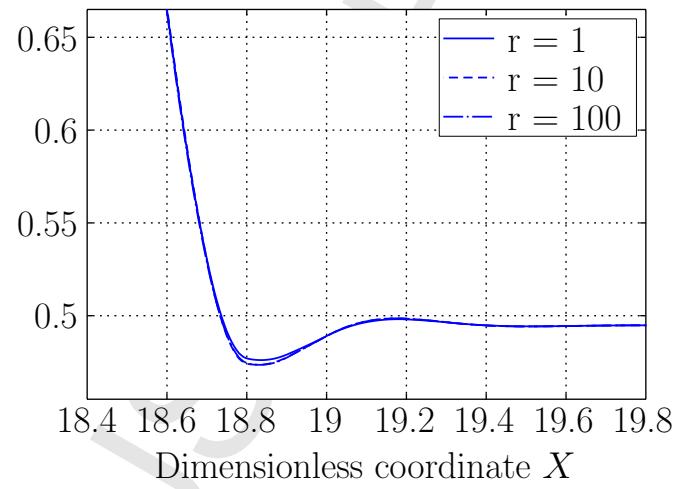

Figure 6: Calculated pressure profiles at time $T=15$, for $\Gamma=0.1, H_{\mathrm{th}}=0.01, w_{\mathrm{cl}}=10, w_{\mathrm{stab}}=$ $0.1, \rho_{\mathrm{cl}}=0.2$ and $l_{e}=0.2$ (left) as well as $l_{e}=0.1$ (right).
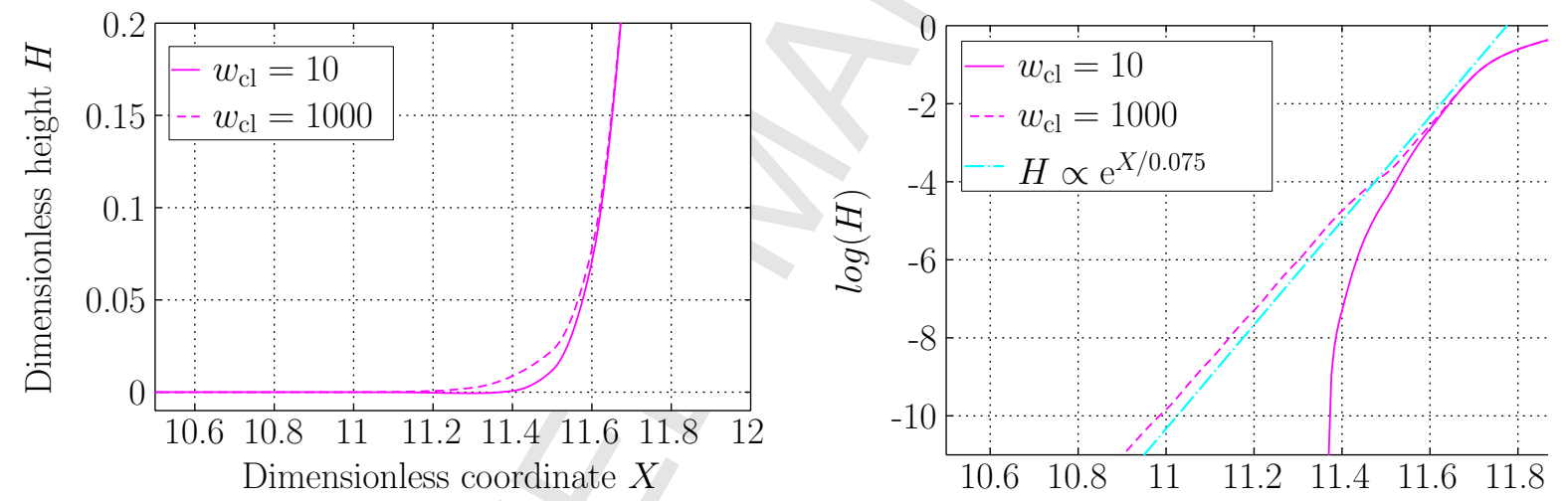

Figure 7: Calculated film thickness profiles at time $T=15$, for $\Gamma=0.1, H_{\text {th }}=0.01, w_{\text {stab }}=0.1$, $\rho_{\mathrm{cl}}=0.2, r=10$ and $l_{e}=0.1$.

for two different meshes. For the coarsest mesh there are visible differences between the calculated film thickness profiles for $w_{\text {stab }}=0.01,0.1$ and 1 . At the same time, a moderate impact on the developed pressure can be observed as well. Though, when the intermediate mesh with $l_{e}=0.1$ is considered, all observed discrepancies between the three cases diminish.

The impact of the regularization parameter $H_{\mathrm{th}}$ on the solution is illustrated in Figure 9, where the 300 elements solution from Figure 5 is compared with the corresponding one for $H_{\text {th }}=0.005$ instead of 0.01. It can be observed that halving $H_{\text {th }}$ affects slightly the pressure profile, while the lubricant film remains closer to the slider surface after it is detached from it in the converging gap region, as one would expect.

One last numerical parameter to be discussed here is the length scale $\rho_{\mathrm{cl}}$ used in the pressure cutoff function from Eq. (46). For the cutoff region to enclose the regularized region at contact lines, where $Q_{\mathrm{cl}}$ is active, the parameter $\rho_{\mathrm{cl}}$ must be larger than $\delta_{\mathrm{cl}} l_{e}$ by a safe margin. This observation motivated the choice of $\rho_{\mathrm{cl}}=0.2$ used in the results presented so far. Figure 10 shows simulation results at the same time instant as in all other cases but for $\rho_{\mathrm{cl}}=0.4$ instead of 0.2. Three different element sizes are represented in the figure which makes it equivalent to Figure 5 presented earlier for the original value of $\rho_{\mathrm{cl}}$. 
150 elements

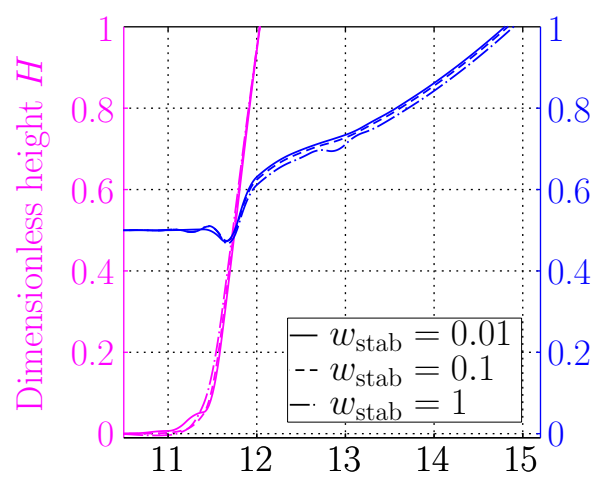

Dimensionless coordinate $X$
300 elements

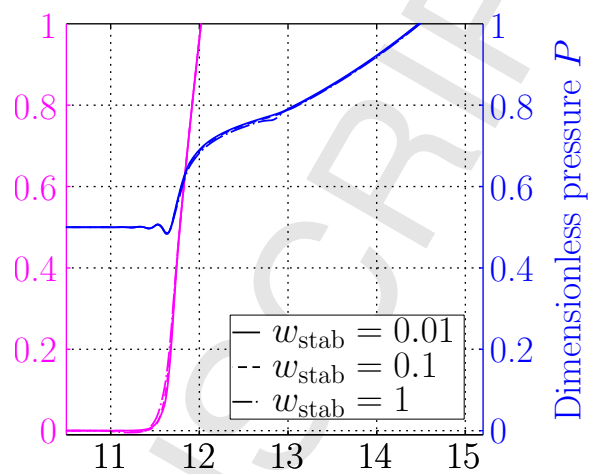

Dimensionless coordinate $X$

Figure 8: Calculated film thickness and pressure profiles at time $T=15$, for $\Gamma=0.1, H_{\mathrm{th}}=0.01$, $w_{\mathrm{cl}}=10, \rho_{\mathrm{cl}}=0.2, r=10$ and $l_{e}=0.2$ (left) as well as $l_{e}=0.1$ (right).
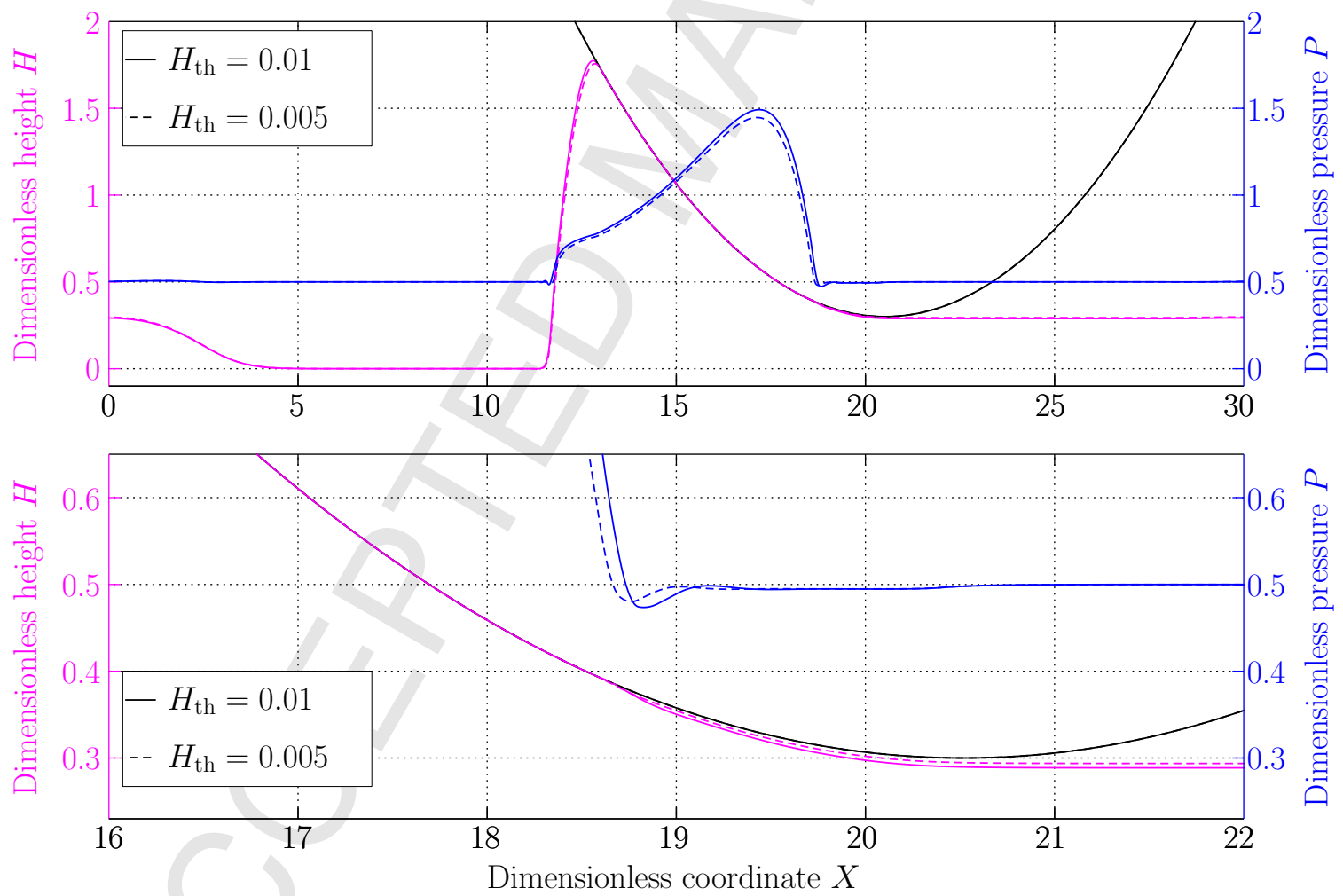

Figure 9: Calculated film thickness and pressure profiles at time $T=15$, for $\Gamma=0.1, w_{\mathrm{cl}}=10$, $w_{\mathrm{stab}}=0.1, \rho_{\mathrm{cl}}=0.2, r=10$ and $l_{e}=0.1$.

Two important observations can be extracted from comparing Figures 5 and 10. The first one regards the much better mesh convergence with respect to the pressure profile in the latter figure, for $\rho_{\mathrm{cl}}=0.4$. This actually indicates that the mesh convergence behavior observed in Figure 5 is actually due to element sizes not being small enough compared to the length scale $\rho_{\mathrm{cl}}$, at least for the coarsest mesh with 150 elements. On the contrary, for the larger $\rho_{\mathrm{cl}}$ value in Figure 10, even 
the 150 elements mesh provides a converged solution with respect to the calculated pressure.

A second important observation regards the obtained maximum hydrodynamic pressures for $\rho_{\mathrm{cl}}=0.2$ and 0.4. Actually, the converged pressure profile in Figure 5 is noticeably higher than the corresponding one for the larger $\rho_{\mathrm{cl}}$ value represented in Figure 10. The reason for this is that a lower length scale parameter $\rho_{\mathrm{cl}}$ corresponds to a lower mobility of lubricant contact lines. In other words, reducing $\rho_{\mathrm{cl}}$ makes contact lines harder to move and favors a pressure buildup in the partially flooded region at the inlet of the hydrodynamic region. This means that the length scale $\rho_{\mathrm{cl}}$ is not simply a regularization parameter but it actually complements the problem definition given in section 2 by providing information about the behavior of contact lines at a phenomenological level. In practice, the pressure cutoff length scale parameter $\rho_{c l}$ can be used to fit experimental data at a given flow rate or corresponding simulations performed at lower size scales. Of course, hard moving contact lines will inevitable require an accordingly fine mesh so that $l_{e}$ will remain safely below $\rho_{\mathrm{cl}} / \delta_{\mathrm{cl}}$ when $\rho_{\mathrm{cl}}$ is set to some very small value.

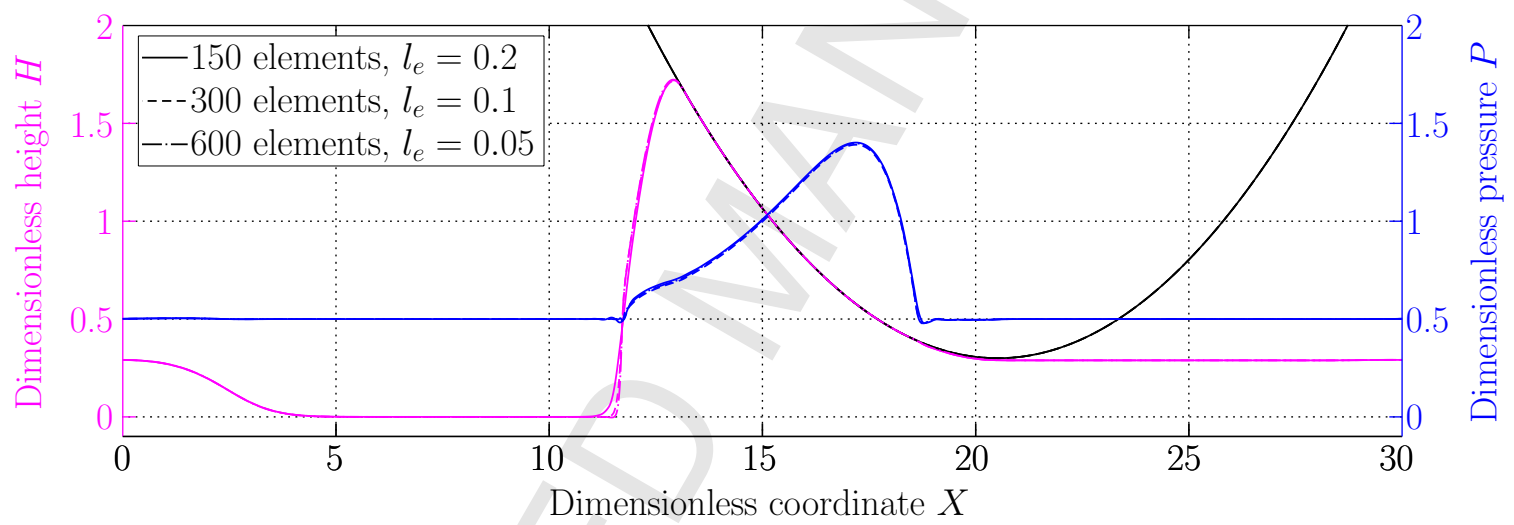

Figure 10: Calculated film thickness and pressure profiles at time $T=15$, for $\Gamma=0.1, H_{\mathrm{th}}=0.01$, $w_{\mathrm{cl}}=10, w_{\mathrm{stab}}=0.1, \rho_{\mathrm{cl}}=0.4, r=10$.

All here presented solutions correspond to a relative displacement of 15 length units between the slider and the substrate with respect to the initial configuration, shown in Figure 4. This arbitrary but representative position corresponds to the 300th time step within a simulation of a total number of 550 time steps, completed without significant convergence issues for all investigated parameter sets and element sizes. The Newton solution of the nonlinear system of equations, required at each time step, typically converged in four iterations irrespective of the element size and the specific set of numerical parameters investigated in this study. The linesearch algorithm, used in combination with the Newton solver, rarely employed scaled Newton steps. In the rare case that the Newton solver with line-search would not converge in individual time steps, as e.g. at the initial onset of contact between the slider and the lubricant film, automatic subdivision of specific steps was sufficient for achieving convergence.

\subsection{Prescribed load simulations}

In all simulation results presented so far, the gap parameter $G$ between the slider and the substrate of Figure 4 has always been considered as prescribed and equal to 0.3. However, prescribing a certain external load instead of the gap $G$ is of higher practical interest in reality. In that case, the scalar quantity $G$ is an additional unknown and a load equilibrium equation has to be introduced 
for determining $G$. This condition can be expressed in variational form as

$$
\int_{\Omega}\left(\xi P_{h}-\bar{P}\right) \mathcal{G} d \Omega=0 \quad \forall \mathcal{G} \in \mathbb{R},
$$

where $\mathcal{G}$ is a virtual variation of $G$ and $\bar{P}$ is a prescribed dimensionless average pressure, corresponding to the external load distributed over the whole domain $\Omega$. The term $\xi P_{h}$ inside the integral represents the pressure within the fully flooded region of the domain $(\xi=1)$, which is the actual load carrying region.

Simulation results presented below were obtained through time integration of Eqs. (39) and (40), including the modifications proposed in section 3.3, coupled with Eq. (51) for determining $G$ for a given load $\bar{P}=2 / 30$. The incorporation of Eq. (51) results in an additional rather dense row in the global stiffness matrix, involving all degrees of freedom of the unknown field $P$ that lie in the fully flooded region.

Figure 11 shows representative results from simulations under prescribed load for two different values of the surface tension parameter $\Gamma$. In order to achieve convergence at the beginning of these simulations, the external load $\bar{P}$ is ramped in the first ten time steps from zero to its nominal value. The two cases included in the figure illustrate the large impact of the surface tension parameter on the obtained film thickness and hydrodynamic pressure. The observed differences also highlight the importance of the present work, as these results cannot be obtained without simulating both the free surface flow in partially flooded regions and the hydrodynamic flow in fully flooded regions.

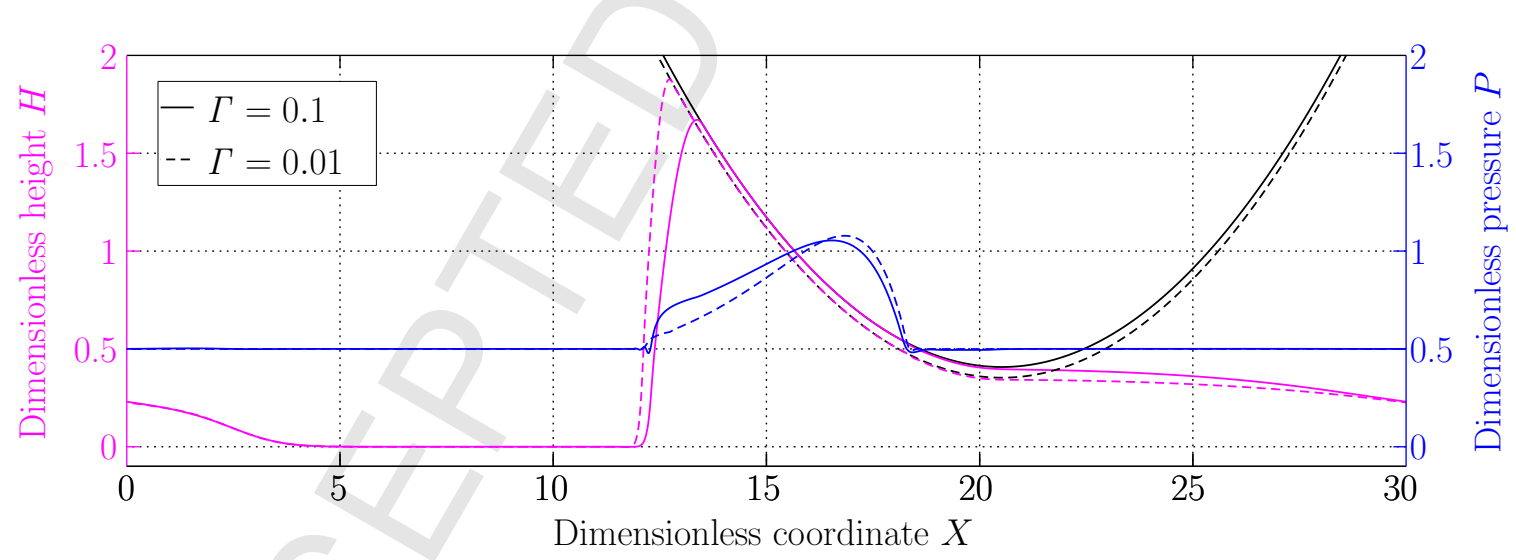

Figure 11: Calculated film thickness and pressure profiles under prescribed load, at time $T=15$, for $H_{\mathrm{th}}=0.01, w_{\mathrm{cl}}=10, w_{\mathrm{stab}}=0.1, \rho_{\mathrm{cl}}=0.2, r=10, l_{e}=0.1$ and $\Gamma=0.1$ as well as $\Gamma=0.01$.

As discussed at the end of section 2, there is some redundancy in providing both the upper and lower solid velocities because it is only the relative velocity between the two solids that governs the flow. This means, that the problem solved so far with $V_{X}=1$ and $\tilde{V}_{X}=0$ is actually equivalent to the case of a stationary substrate, $V_{X}=0$, and a moving slider with a negative velocity $\tilde{V}_{X}=-1$. For the periodic boundary conditions that have been assumed in the investigated example, this equivalence is very easy to confirm numerically. Figure 12 shows results equivalent to the case of $\Gamma=0.1$ from Figure 11 but obtained in a frame of reference that is fixed with respect to the substrate. The differences between the two results, obtained in different frames of reference are only minor. 


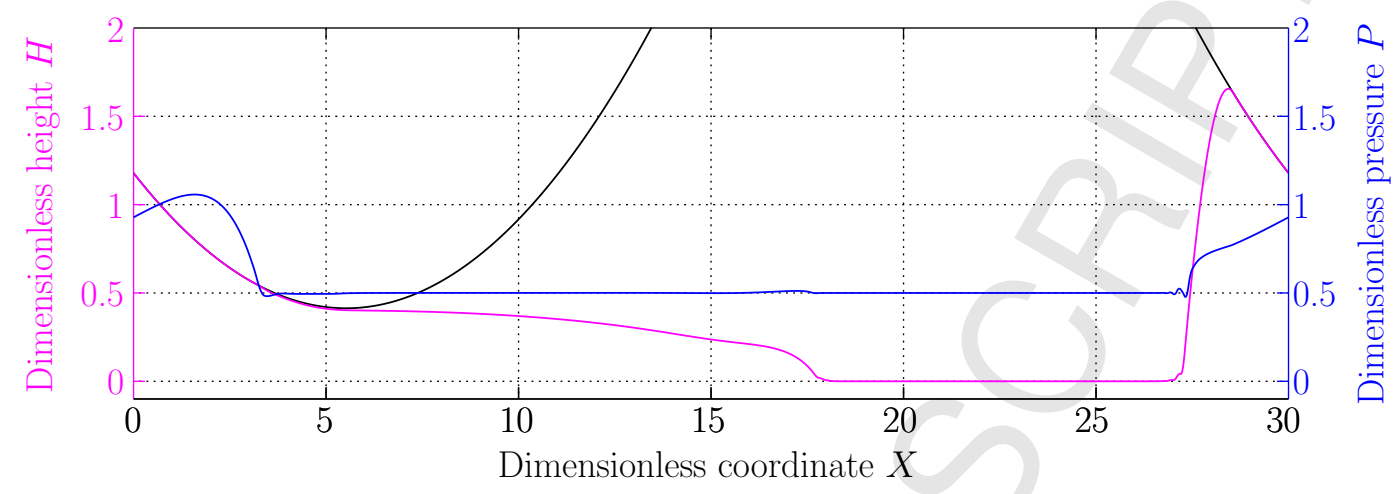

Figure 12: Calculated film thickness and pressure profiles under prescribed load, at time $T=15$, for $H_{\mathrm{th}}=0.01, w_{\mathrm{cl}}=10, w_{\mathrm{stab}}=0, \rho_{\mathrm{cl}}=0.2, r=10, l_{e}=0.1, \Gamma=0.1$ (frame of reference fixed to the substrate).

\section{Concluding remarks}

The developed finite element method unifies the simulation of fully flooded hydrodynamic flow and partially flooded thin film free surface flow, without involving an explicit resolution of the boundary between the two domains. The two sets of equations to be solved respectively in fully and partially flooded regions were formulated as a complementarity problem similar to the augmented Lagrangian method for modeling unilateral contact in structural mechanics. A strong discontinuity in the lubricant top surface velocity at the boundary between the two domains was treated through regularization involving a corresponding regularization parameter. In a further step, the resulting inequality constrained partial differential equations were replaced through equivalent unconstrained but only piecewise smooth equations, discretized by means of a weighted residual approach. In the final global system of equations, dimensionless versions of both the film thickness $h$ and the pressure $p$ were retained as unknowns.

Regarding the discretization of the obtained equations, the presence of second order derivatives required a $\mathrm{C}^{1}$ continuous approximation of the film thickness field. In that regard, the present work actually proposes the use of quadratic B-spline basis functions both for the film thickness and pressure fields, which proved successful in the presented numerical tests.

Some special treatment was necessary for the film contact lines, namely the boundaries between wetted and dry regions. A novel method for preserving non-negativity of the solution was proposed based on an additional flow term that becomes non-zero only close to contact lines. Moreover, a nonlinear stabilization for advection terms was proposed specifically for $\mathrm{C}^{1}$ continuous approximations. Last, a length scale parameter was introduced for deactivating capillary effects close to contact lines and its value was found to govern the mobility of contact lines. Numerical studies in an one dimensional domain showed that all further numerical parameters introduced in the derivation of the proposed model do not affect the obtained results within a wide range of values.

The presented numerical results have also demonstrated the significant role of the surface tension parameter not only in the partially flooded zone but also in the hydrodynamic zone, through the inevitable coupling of the two. This contribution highlights the relevance of the proposed numerical method for the simulation of non-conformal hydrodynamic contacts, where a hydrodynamic contact is surrounded by free surface flow regions, such as the contact between a piston ring and a cylinder liner in internal combustion engines. 
From a numerical perspective, the non-negativity preserving formulation proposed in the present work is expected to be useful in a broader context than the specific lubrication problem dealt with here, possibly also in connection to standard Lagrange elements. Moreover, the proposed nonlinear stabilization scheme for advection terms, although specific to $\mathrm{C}^{1}$ continuous elements, it has the potential of a more general application within the emerging field of approximations with higher degree of continuity.

\section{Acknowledgements}

This work was supported by the European Commission under the Hercules-2 project, Fuel Flexible, Near Zero Emissions, Adaptive Performance Marine Engine (Grant Agreement no 634135HERCULES-2).

\section{References}

Bertozzi, A. L. (1998). The mathematics of moving contact lines in thin liquid films. Notices of the American Mathematical Society, 45(6):689-697.

Bertozzi, A. L. and Bowen, M. (2002). Thin film dynamics: theory and applications. Nato Science Series II: Mathematics, Physics and Chemistry, 75:31-79.

Bornemann, P. B. and Cirak, F. (2013). A subdivision-based implementation of the hierarchical b-spline finite element method. Computer Methods in Applied Mechanics and Engineering, 253:584-598.

Cryer, C. W. (1971). The method of Christopherson for solving free boundary problems for infinite journal bearings by means of finite differences. Mathematics of Computation, 25(115):435-435.

Curnier, A. and Alart, P. (1988). A generalized Newton method for contact problems with friction. Journal de Mecanique Theorique et Appliquee, 7(1):67-82.

de Gennes, P. (1985). Wetting: statics and dynamics. Rev. Mod. Phys., 57:827-863.

Dowson, D. (1962). A generalized Reynolds equation for fluid-film lubrication. International Journal of Mechanical Sciences, 4:159-170.

Dowson, D. and Taylor, C. M. (1979). Cavitation in bearings. Annual Review of Fluid Mechanics, 11(1):35-66.

Elrod, H. G. (1981). A cavitation algorithm. Journal of Lubrication Technology, 103(3):350-354.

Kostreva, M. M. (1984). Elasto-hydrodynamic lubrication: A non-linear complementarity problem. International Journal for Numerical Methods in Fluids, 4(4):377-397.

Organisciak, M., Cavallaro, G., and Lubrecht, A. A. (2007). Variable lubricant supply of a starved hydrodynamic linear contact: Lubricant lateral flow for smooth and laser textured surfaces. Proceedings of the Institution of Mechanical Engineers Part J: Journal of Engineering Tribology, 221(J3):247-258.

Pommier, J. and Renard, Y. (2017). GetFEM++, an open source generic C++ library for finite element methods. http://getfem.org/. 
Reynolds, O. (1886). On the theory of lubrication and its application to Mr. Beauchamp Tower's experiments, including an experimental determination of the viscosity of olive oil. Philosophical Transactions of the Royal Society of London, 177(0):157-234.

Rohde, S. M. and McAllister, G. T. (1975). Variational formulation for a class of free boundary problems arising in hydrodynamic lubrication. International Journal of Engineering Science, 13(9-10):841-850.

Strozzi, A. (1985). Formulation of three lubrication problems in terms of complementarity. Wear, 104(104):103-119.

Stupkiewicz, S., Lengiewicz, J., Sadowski, P., and Kucharski, S. (2016). Finite deformation effects in soft elastohydrodynamic lubrication problems. Tribology International, 93:511-522.

Yang, B. and Laursen, T. A. (2009). A mortar-finite element approach to lubricated contact problems. Computer Methods in Applied Mechanics and Engineering, 198(47-48):3656-3669.

Zhang, S., Wang, W., Li, X., and Guo, F. (2016). The effect of oil droplet on the lubrication performance. Journal of Tribology, 138(3):031506.

Zhornitskaya, L. and Bertozzi, A. L. (2000). Positivity-preserving numerical schemes for lubrication-type equations. Siam Journal on Numerical Analysis, 37(2):523-555. 